\title{
Is successful HIV therapy a Pyrrhic victory for the brain?
}

\author{
David B. Clifford
}

Department of Neurology, Washington University, St. Louis, Missouri, USA

\begin{abstract}
Neurologic involvement of HIV remains an important concern for patients, physicians, and investigators. Catastrophic decline is rarely seen in patients on combination antiretroviral therapy (CART); however, neurological decline remains a critical clinical challenge. In this issue of the $J C I$, Spudich and associates investigated the status of HIV in the cerebral spinal fluid (CSF) and revealed ongoing presence of HIV in the nervous system. Surprisingly, even in the face of optimal treatment, including suppressed HIV RNA, almost half of the patients investigated showed cell-associated HIV (CAHIV) DNA in the CSF. Spudich et al. find that persistence of HIV in CSF cells is associated with lower performance on neurocognitive testing. These findings emphasize the need to consider a viral-associated mechanism as playing a significant and potentially ongoing role in HIV-associated neurocognitive disorder (HAND).
\end{abstract}

of successful HIV therapy is a Pyrrhic, or hollow, victory.

The most striking finding in Spudich et al. - that such a large proportion of patients have cells harboring HIV DNA - raises several critical questions that are important but technically difficult to answer (4). What cell type contains this residual virus remains a key question. Spudich and associates used an age-matched neurologically normal HIV-negative volunteer population to compare CSF cell counts in the setting of well-treated HIV. It is remarkable that CSF cell counts are essentially normal in those with welltreated HIV. While the profile may reflect normal nucleated cell populations in CSF with lymphocytes, monocytes, B cells, and NK cells, the affected cells could even represent a unique brain-associated population that may migrate to the CSF compartment (5). Future studies might explore a variety of questions. Are the cells containing HIV DNA simply part of the ongoing trafficking of cells entering and leaving the brain that are involved in routine immunological monitoring of this critical organ? Could these cells possess a cytokine expression profile that promotes their transfer into the CSF? Are the viral copies found in the CSF cells replication competent? What is the relation between the sequences of HIV in these cells and those in peripheral and CNS locations? Emerging technologies such as single-cell RNA sequencing could explore the expression patterns represented in CSF cells of patients with and without CA-HIV DNA or HAND to expand the understanding of this critical cohort of cells (5).

\section{The role of inflammation and HIV in neurological deterioration}

Spudich et al. report concurrent inflammatory markers in CSF to consider mechanisms of potential ongoing injury (4). The findings challenge the hypothesis that HIV-associated brain dysfunction is driven by immune activation. Lacking simpler explanations, an inflammatory mechanism probably stimulated by residual HIV has 
too often been presented as the most likely cause of ongoing neurological involvement in HIV patients (6). Lack of correlation of inflammatory markers with viral presence in the CSF in this study, as well as with neurocognitive performance, brings this hypothesis into question in a way that should be healthy for the field. If residual virus triggering ongoing inflammation is the mechanism for neurocognitive impairment, therapeutic efforts such as changing therapy to intensify or increase braindrug penetrance are rational approaches to manage HAND $(7,8)$. Based on this study, it is not surprising that these strategies may fail to meaningfully change the inflammatory profile and perhaps the neurocognitive deficits.

Chronic immune activation in HIV patients remains a valid observation but whether it is truly sufficient to drive ongoing brain dysfunction or damage is completely unproven. The data presented by Spudich et al. do not support the hypothesis that residual HIV within the brain compartment drives this immune activation. More longitudinal analysis of CSF inflammatory responses and residual HIV in the CNS are needed to critically evaluate the chronic smoldering inflammatory response on neurocognitive performance. Further studies could compare HIV-treated patients to patients with other diseases, such as inflammatory bowel disease, that result in chronic immune activation.

Some contend that HIV results in accelerated aging related to degeneration of brain physiology and structures. However, neurocognitive performance gaps may accrue early in infection, then evolve in parallel with normal age-related changes. Successfully treated HIV patients (in whom it seems CA virus is prevalent) have a similar aging pace as the general population in some but not all studies (9-12). In part because the HIV population has complex comorbidities that contribute and likely dominate the impact of HIV itself, assembling high-quality longitudinal, well-controlled patient studies has been difficult. Notably, many of the same fac- tors driving declining function in the general population deserve immediate attention in our HIV patients; most salient are those that impact vascular disease (including diabetes, smoking, blood pressure control, and cholesterol) (13-15). Given the decrement in function that often occurs in HIV patients, addressing vascular factors and helping our patients adopt a healthy, active lifestyle may yield more fruitful neuroprotection than changes in traditional viral-directed interventions (16).

It is important not to catastrophize the results of this study. Spudich et al. report that patients both with and without CSF CA-HIV DNA had median neurocognitive performances in the normal range (4). While the percentage of participants whose performance fell in the impaired range was higher for those with virus in the CSF, the difference in performance was notably modest. The small cross-sectional difference between the two groups leaves room for questioning whether the virus underlies this change. Longitudinal measures will be critical to assess the relationship between cognitive function and CA-HIV DNA in the nervous system. The surprising prevalence of viral DNA in cells in the CSF revealed by Spudich et al. justifies continued investigation into how HIV might directly impact brain function in treated HIV patients, and begs discovery of practical ways to eliminate the virus from this reservoir.

\section{Acknowledgments}

The author gratefully acknowledges the many generous patients participating in his trials and clinic, his colleagues, and the support of NIH grants AI069439, NS10719801, MH10734504, AG0617904, AG05326702, and UL1TR00234502.

Address correspondence to: David B. Clifford, Box 8111, 660 South Euclid Avenue, Saint Louis, Missouri 63110, USA. Phone: 314.747.8423; Email: clifforddb@wustl.edu.

1. Navia BA, Jordan BD, Price RW. The AIDS dementia complex: I. Clinical features. Ann Neurol. 1986;19(6):517-524.

2. Heaton RK, et al. HIV-associated neurocognitive disorders persist in the era of potent antiretroviral therapy: CHARTER Study. Neurology. 2010;75(23):2087-2096.

3. Heaton RK, et al. Neurocognitive change in the era of HIV combination antiretroviral therapy: the longitudinal CHARTER study. Clin Infect Dis. 2015;60(3):473-480.

4. Spudich S, et al. Persistent HIV-infected cells in cerebrospinal fluid are associated with poorer neurocognitive performance. JClin Invest. 2019;129(8):3339-3346.

5. Farhadian SF, et al. Single-cell RNA sequencing reveals microglia-like cells in cerebrospinal fluid during virologically suppressed HIV. JCI Insight. 2018;3(18):121718.

6. Klatt NR, Chomont N, Douek DC, Deeks SG. Immune activation and HIV persistence: implications for curative approaches to HIV infection. Immunol Rev. 2013;254(1):326-342.

7. Letendre S, et al. Validation of the CNS penetration-effectiveness rank for quantifying antiretroviral penetration into the central nervous system. Arch Neurol. 2008;65(1):65-70.

8. Ellis RVF, et al. A randomized, controlled trial of a central nervous system-targeted ART strategy for HIV-associated neurocognitive disorders. 20th Conference on Retroviruses and Opportunistic Infections - Atlanta, GA. March, 2013. http://www.natap.org/2013/ CROI/croi_144.htm. Accessed July 3, 2019.

9. Cole JH, et al. Increased brain-predicted aging in treated HIV disease. Neurology. 2017;88(14):1349-1357.

10. Sanford R, et al. Longitudinal trajectories of brain volume and cortical thickness in treated and untreated primary human immunodeficiency virus infection. Clin Infect Dis. 2018;67(11):1697-1704.

11. Ances BM, et al. HIV infection and aging independently affect brain function as measured by functional magnetic resonance imaging. J Infect Dis. 2010;201(3):336-340.

12. Janssen MAM, et al. Resting-state subcortical functional connectivity in HIV-infected patients on long-term cART. Brain Imaging Behav. 2017;11(5):1555-1560.

13. Yuen T, et al. Personalized risk index for neurocognitive decline among people with well-controlled HIV infection. J Acquir Immune Defic Syndr. 2017;76(1):48-54.

14. Wright EJ, et al. Cardiovascular risk factors associated with lower baseline cognitive performance in HIV-positive persons. Neurology. 2010;75(10):864-873.

15. Monnig MA, et al. Effects of smoking and alcohol use on neurocognitive functioning in heavy drinking, HIV-positive men who have sex with men. AIDS Care. 2016;28(3):300-305.

16. Dufour CA, et al. Physical exercise is associated with less neurocognitive impairment among HIV-infected adults. J Neurovirol. 2013;19(5):410-417. 